\title{
In-situ synchrotron tomography estimation of toughening effect by semi-ductile fibre reinforcement in a tungsten fibre-reinforced tungsten composite system
}

\author{
J. Riesch ${ }^{\mathrm{a}}$, J.-Y. Buffière ${ }^{\mathrm{b}}$, T. Höschen ${ }^{\mathrm{a}}$, M. di Michiel ${ }^{\mathrm{c}}$, M. Scheel ${ }^{\mathrm{c}}$, Ch. Linsmeier ${ }^{\mathrm{a}}$, J.-H. You ${ }^{\mathrm{a}, *}$ \\ ${ }^{a}$ Max-Planck-Institut für Plasmaphysik, EURATOM Association, 85748 Garching, Germany \\ ${ }^{b}$ GEMPPM INSA Lyon, 20 Av. A. Einstein, 69621 Villerbanne, Cedex, France \\ ${ }^{c}$ European Synchrotron Radiation Facility, BP 220, 38043 Grenoble, France
}

\begin{abstract}
Tungsten-fibre reinforced tungsten composites $\left(W_{f} / W\right)$ are supposed to enable enhanced toughness owing to extrinsic energy dissipation mechanisms such as interface debonding and plastic deformation of fibre. In particular, the latter is an effective source of toughening, since ductile tungsten fibre can absorb considerable amount of plastic work. For a precise evaluation of the toughening capability, the energy dissipation mechanisms need to be analysed in detail. To this end, single-fibre tungsten composite specimens are fabricated and the stress-strain behaviour of the tungsten fibre bridging a matrix crack is measured by means of in-situ high energy synchrotron micro-tomography during an uniaxial tension test. Despite the high X-ray attenuation in tungsten a sufficiently high resolution is achieved and clear images of crack extension and deformation are obtained. The amount of absorbed energy due to plastic deformation of the tungsten fibre is determined and compared with values obtained conventionally from single-fibre tension tests.
\end{abstract}

Keywords: tungsten, fibre-reinforced composite, toughness, synchrotron tomography, in-situ tension test

\section{Introduction}

Due to its unique combination of desired properties, tungsten is the most favoured candidate material for the plasmafacing components of fusion reactors [1]. However, the inherent brittleness of tungsten below the ductile-to-brittle transition temperature is a critical concern. Furthermore, embrittlement due to recrystallization or neutron irradiation may strongly restrict the applicability of tungsten in plasma-facing components $[1,2]$. Metallurgical efforts to overcome such an essential limitation have been focused on the enhancement of ductility via microstructural refinement or mechanical alloying of dispersed particles, but the state-of-the-art of these approaches is still far from the design requirements. Comprehensive reviews on this topic can be found in the literature $[3,4]$.

Recently, authors proposed a new toughening concept for tungsten, where tungsten is reinforced by tungsten wires with an engineered interface $[5,6,7,8]$. In this tungsten fibrereinforced tungsten composite $\left(W_{f} / W\right)$, the toughness is enhanced by extrinsic mechanisms of energy dissipation, which is called extrinsic toughening in the following. This toughening mechanism is widely applied in fibre-reinforced ceramic matrix composites $[9,10]$. The deformation energy stored in the composite is mostly dissipated through the debonding and frictional sliding at fibre/matrix interfaces while matrix cracks are bridged by the fibres being pulled out. These dissipative processes lead to considerable energy absorption accompanied by mitigation of stress intensity near a crack tip. As the underlying mechanism is based on a purely mechanical effect, it should

${ }^{*}$ Corresponding author: jeong-ha.you@ipp.mpg.de be hardly affected by additional embrittlement in fusion reactor environment.

In the case of $W_{f} / W$ composites, an additional effective source of energy dissipation is available, i.e. plastic deformation of the tungsten fibres. Tungsten wires, fabricated by a drawing process, are capable of plastic deformation and can undergo tensile elongation up to several percent. Thus, the plastic work conducted by semi-ductile tungsten fibres is an additional, presumably considerable contribution to the toughening of $W_{f} / W$ composites. For a quantitative estimation of the amount of energy absorbed by the plastic work of tungsten fibres, the actual stress-strain behaviour of the constrained fibres has to be measured under a realistic loading condition [11].

In this paper, we present a tomography-based method for direct observation of fibre deformation and interface debonding. Since this technique enables in-situ observation of the deformation and fracture process during loading, the progress of plastic work can be quantified as a function of the applied load. The strong X-ray absorption of tungsten on the one hand and the need for in-situ experiments on the other hand require high energy synchrotron radiation for the tomographic measurements. Only with high-energy X-rays both a sufficient sample penetration and a fast data acquisition (fast tomography) are feasible. However, the trade-off between energy and spatial resolution in X-ray tomography raises technical challenges for the high $\mathrm{Z}$ metal tungsten. In the following we describe the results of an experiment performed at ESRF on beamline ID-15A. A tomography-based methodology for assessing the plastic work by fibre deformation is presented. The results are compared to those of single-fibre tension tests on free fibres. Finally, the 
contribution of plastic fibre deformation to the toughening of a multi-fibre composite system is estimated.

\section{Toughening by the plastic work of fibre deformation}

Chemically deposited tungsten used as matrix material in the $W_{f} / W$ composite shows brittle behaviour [12] and can therefore be described by linear elastic fracture mechanics. In such materials toughness and therefore the contribution of fibre reinforcement can be expressed in terms of energy release rate $G$, which is defined as the change in elastic potential energy per unit area of an extending crack. If $G$ is higher than the critical value $G_{\mathrm{c}}$, then the material will fail. Thus, $G_{\mathrm{c}}$ (fracture energy) is a measure of material's resistance against brittle fracture [13].

For the $W_{f} / W$ composite, the total toughness consists of two contributions:

$$
G_{\mathrm{c}}=G_{0}+\Delta G
$$

where $G_{0}$ denotes the fracture energy in the absence of reinforcement and $\Delta G$ is the increment of fracture energy due to the extrinsic toughening effect. The amount of plastic work to be dissipated depends on the stress between the crack surfaces bridged by a tungsten fiber and its evolution in time. For a single crack, the plastic work per unit area of crack face, $\Delta G_{\mathrm{pl}}$, is given by $[14,11]$ :

$$
\Delta G_{\mathrm{pl}}=V_{\mathrm{f}} \cdot w_{\mathrm{pl}}^{*}=V_{\mathrm{f}} \int_{0}^{u_{\max }} \sigma(u) \mathrm{d} u
$$

with $V_{\mathrm{f}} \quad$ : volume fraction of reinforcements $w_{\mathrm{pl}}^{*}:$ specific plastic deformation energy $u_{\max }$ : maximum crack opening $\sigma(u):$ stress distribution in reinforcements

$[-]$ $\left[\mathrm{J} \mathrm{m}^{-2}\right]$ $[\mathrm{m}]$ [MPa]

Assuming that the fibre yield stress increases linearly with tensile strength, and further that the ultimate plastic strain is proportional to the fibre radius, Ashby suggested the following empirical relationship between the toughness increase and the plastic behaviour of the reinforcing fibre [11]:

$$
\Delta G_{\mathrm{pl}}=V_{\mathrm{f}} \cdot w_{\mathrm{pl}}^{*}=V_{\mathrm{f}} \chi \sigma_{\mathrm{e}} r_{\mathrm{f}}
$$

where

$$
\chi=\frac{w_{\mathrm{pl}}^{*}}{\sigma_{\mathrm{e}} r_{\mathrm{f}}}=2,5 \cdot \frac{u_{\mathrm{max}}}{r_{\mathrm{f}}}
$$

with

$$
\begin{array}{ll}
\chi: \text { toughening parameter } & {[-]} \\
\sigma_{\mathrm{e}}: \text { yield strength } & {[\mathrm{MP}} \\
r_{\mathrm{f}}: \text { radius of reinforcement } & {[\mathrm{m}]}
\end{array}
$$

Equation 3 gives a linear relation between the normalised deformation energy $w_{\mathrm{pl}}^{*} / \sigma_{\mathrm{e}} r_{\mathrm{f}}$ and the normalised crack opening $u_{\max } / r_{\mathrm{f}}$.

Ashby demonstrated also that metal wires, which are embedded and constrained in a brittle matrix (in such a manner that deformation is restricted to a small segment of the wire relaxed by a matrix crack), show force-displacement curves significantly deviating from those of an unconstrained counterpart.

The amount of plastic deformation energy of a reinforcing fibre, which makes a major contribution to toughness, depends strongly on the development of the plastic zone in the fibre and thus on the extent of interface debonding. Here, one can distinguish three cases: extensive, intermediate, or negligible debonding. In the case of negligible debonding, the fibre remains fully constrained and the restricted plastic zone allows only limited energy dissipation. If debonding occurs extensively, the load transfer between fibre and matrix becomes no more effective and almost no energy is being dissipated. The intermediate case allows more or less energy dissipation depending on the debonding length.

In order to accurately estimate the magnitude of $\Delta G_{\mathrm{pl}}$, the actual stress-strain behaviour (i.e. in the measure of true stress and true strain) of the tungsten fibre constrained in a composite is needed. This information can be extracted from an in-situ tomography measurement which produces data of the current configuration of the local geometry. The quantities of interest are the plastic elongation of fiber (expressed as crack opening $u$ ) over the debonded interface (expressed as debonding length $u_{\mathrm{db}}$ ) and the reduction of the fibre cross section. The total plastic work is then obtained by integrating all work increments estimated at individual tomography steps (corresponding to loading steps) during an uniaxial tensile test. The in-situ information of the extent of the debonding as well as its temporal variation is important in order to understand the influence of the constraint.

\section{Experiment}

\subsection{Specimen preparation}

For an in-situ measurement of the fibre deformation the use of a single-fibre-reinforced composite offers simpler and clearer observations than a many-fibre counterpart. In this case the plastic work to be estimated for the composite system is automatically a normalized quantity for a single-fibre case. Therefore a model composite system, consisting of a single tungsten fibre embedded in a tungsten matrix is fabricated. For the fibre a commercial tungsten wire provided by Osram $\mathrm{GmbH}$, Schwabmünchen is used. This wire has a high strength (> $2500 \mathrm{MPa}$ ) and a good ductility (failure strain $>2 \%$ ). The wire diameter in the composite is $150 \mu \mathrm{m}$ whereas in the single fibre tension tests wires with a diameter of $75 \mu \mathrm{m}$ and $125 \mu \mathrm{m}$ are tested. The tungsten matrix is deposited by means of chemical vapour deposition (CVD). Prior to the matrix deposition, the fibre surface is coated with a tungsten oxide film (thickness: $0.5 \mu \mathrm{m}$ ) to form an engineered fibre/matrix interface having optimal fracture energy. As the temperature during the CVD processing is moderate for tungsten material (about $550{ }^{\circ} \mathrm{C}$ ), the fine microstructure and strong texture of the embedded tungsten fibres is preserved. A detailed description of the fabrication technology is given elsewhere [15].

In Figure 1, metallographic cross-sections of the single-fibre composites are shown. The picture on the left hand side shows a cross-section transverse to the fibre axis through the whole specimen. The fibre is located in the centre surrounded by the matrix having a radial grain structure. On the right hand side the interface between fibre and matrix is shown in detail. The longitudinal grain structure of the fibre can clearly be distinguished from the more isotropic structure of the matrix. For 


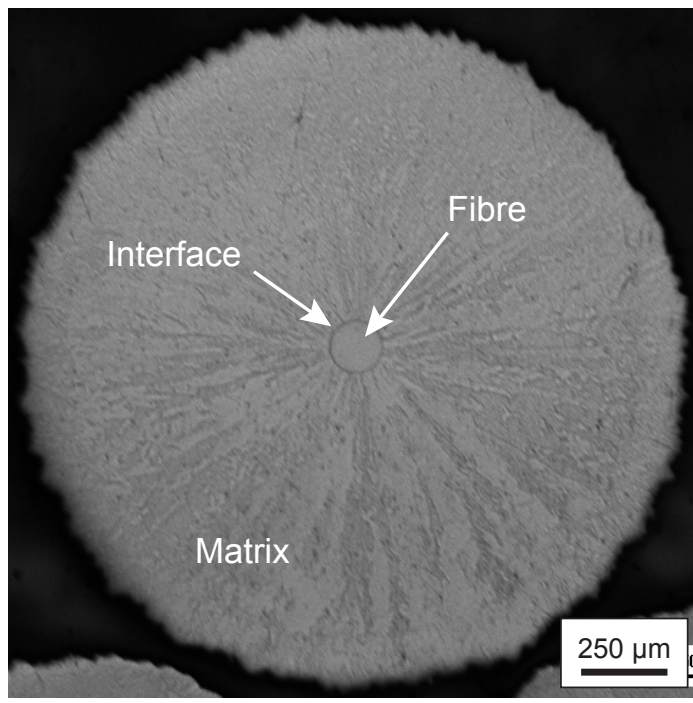

(a)

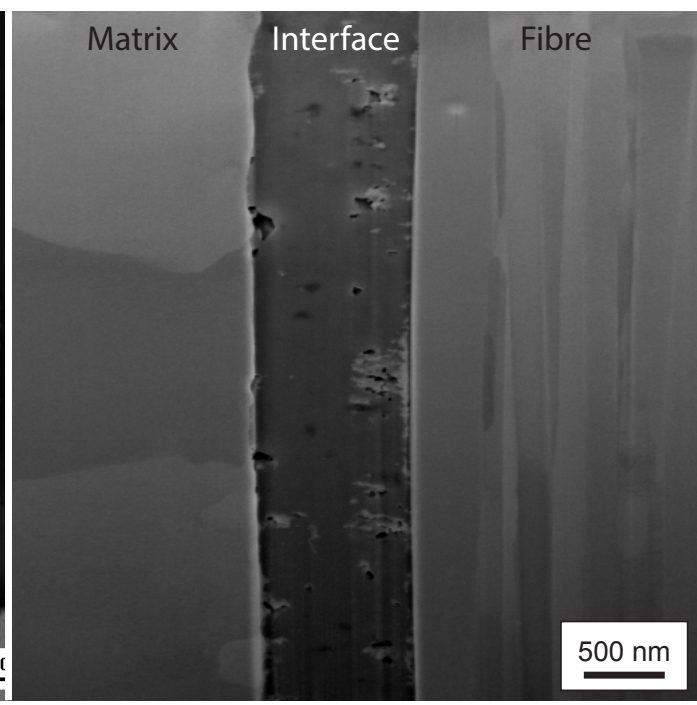

(b)

Figure 1: Metallographic cross sections of a single fibre $W_{\mathrm{f}} W$ composite consisting of a single coated tungsten wire which is embedded in a tungsten matrix formed by chemical vapour deposition. In (a) a cross section of the whole sample is shown. In (b) a cross section along the fibre axis shows the interface between fibre and matrix in detail. The preserved longitudinal grain structure in the fibre is well visible.

the tomographic investigation, cylindrical specimens are produced in order to maximize the specimen volume for a given maximum penetration depth. In the CVD process cylindrical samples with a diameter of $1.5-2.2 \mathrm{~mm}$ are produced. These samples are subsequently machined to a $1 \mathrm{~mm}$ diameter in the central area. Afterwards they were attached to aluminium tabs using a two component epoxy resin yielding dog-bone shaped samples (see Figure 2). The samples are notched using a wire saw $(80 \mu \mathrm{m}$ wide and $100 \mu \mathrm{m}$ deep). The actual pre-crack is created at the notch root using a focused ion beam ( $1 \mu \mathrm{m}$ wide and $10 \mu \mathrm{m}$ deep).

\subsection{Tomography}

For the in-situ observation of internal deformation and failure occurring in tungsten materials, high-energy synchrotron tomography is the most adequate technique. High energy X-ray synchrotron radiation allows tomography imaging of optically opaque materials with a spatial resolution in the range of a few microns [16]. In case of the strongly absorbing tungsten the critical issue is to achieve a sufficient penetration depth. Recently, in the framework of FEMaS program, the authors conducted a series of experimental campaigns at the high energy beamline ID15-A at the European Synchrotron Radiation Facility (ESRF) in Grenoble and established the technical conditions for tungsten tomography up to a penetration depth of $1 \mathrm{~mm}$ [17]. This successful preparatory beamline calibration allows now to accurately trace the deformation and fracture processes in the single-fibre $W_{f} / W$ composites. From the computed tomography the instantaneous changes in the sample geometry during the in-situ tension tests can be observed.

Tomography of $W_{f} / W$ composite is quite challenging due to the strong attenuation of X-rays in tungsten [18]. For a sample thickness of $1 \mathrm{~mm}$, the intensity of transmitted X-rays is

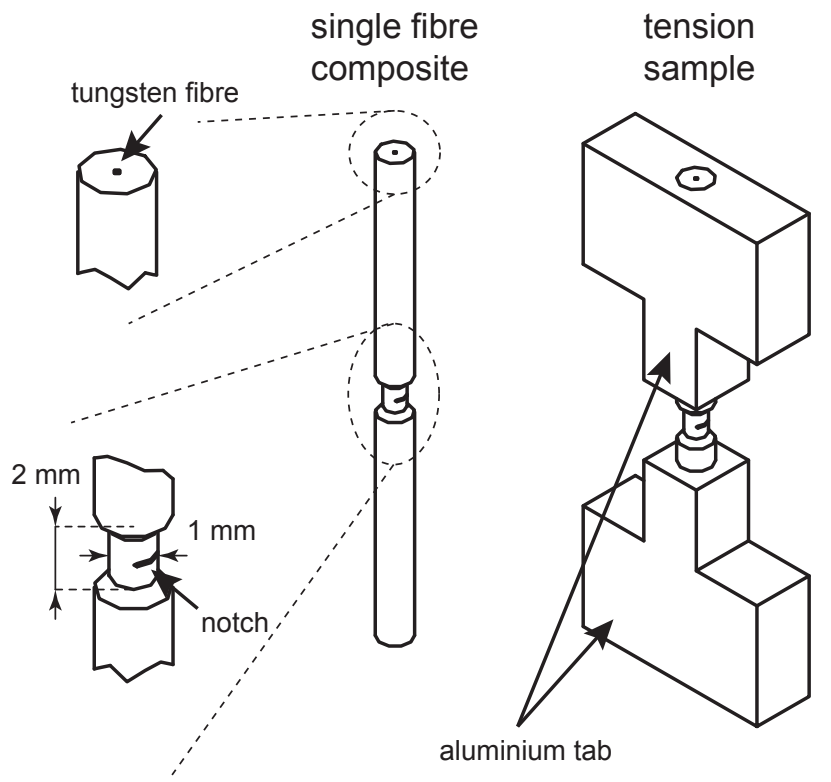

Figure 2: 3D-sketch of dog-bone shaped tension samples consisting of a single fibre composite attached to aluminium tabs by gluing. 
reduced by $97 \%$ whereas the reduction is $70 \%$ for a thickness of $0.25 \mathrm{~mm}$. These values show that a thickness increase of the tungsten specimens causes a rapid decrease of X-ray intensity available for tomographic imaging. Moreover, there is a limitation in reducing the specimen thickness to avoid dominating effects from the limited size, e.g. the free surface effects becoming dominant in the mechanical behaviour of a composite. In addition practical reasons like the difficult machining of brittle CVD tungsten specimens becomes limiting. A further technical constraint is the trade-off between spatial resolution and photon energy [19]. It is impossible to increase the penetration depth with higher X-ray energies without degrading the quality of the tomographic reconstructions. Therefore, although a larger sample thickness is advantageous for reliability of the test data, the optimal compromise between sample thickness, tomographic resolution and speed of measurement has to be used.

In the present study, we chose a white beam with an energy of $60-250 \mathrm{keV}$ and a sample diameter of $1 \mathrm{~mm}$. This combination allows a sufficiently fast tomography measurement. For one tomogram 3600 tomographic projections with an exposure time of $80 \mathrm{~ms}$ each are collected over a rotation angle of $180^{\circ}$. The total acquisition time for a full tomogram is approximately 10 min. A LuAG:Ce scintillator with a thickness of $25 \mu \mathrm{m}$, twostep optical lenses (total magnification 10 times) and a Snarnoff CAM512 CCD-camera (512x512 pixels) are used. The final spatial resolution achieved is approximately $5 \mu \mathrm{m}$ (voxel size: $\left.1.9 \mu \mathrm{m}^{3}\right)$. The image reconstruction is carried out by filtered back-projection. For the 3-dimensional image analysis the software ImageJ/Fiji [20] is used. The round geometry of the cylindrical specimens caused premature camera saturation at its peripheral domain. To solve this problem, a lead aperture disc is used which is thicker at the outer part of the specimens so that the nonuniform transmission along the radius is compensated.

\subsection{Tensile test}

The single fibre tension tests (without contstraint) are conducted in a displacement controlled mode using a universal testing machine (TIRA Test 2820). The displacement is measured by a laser-speckle-extensonmeter (LSE 4000-DE), the load is detected by a $200 \mathrm{~N}$ load cell.

For in-situ tensile testing, a dedicated tensile machine is installed into the synchrotron radiation detection system. The maximum load is $5 \mathrm{kN}$ with a minimal displacement rate of $0.1 \mu \mathrm{m} \mathrm{s}^{-1}$. A detailed description on the machine is given elsewhere [21]. The tension tests are carried out in a displacementcontrolled mode. A tomogram is produced at each displacement increment under stepwise tensile elongation.

\section{Results and discussion}

Figure 3 shows the result of the single fibre tension tests on fibres with two different diameters. Both fibres show similar behaviour: after an elastic deformation, stress levelling occurs until a maximum is reached. After a slight stress decrease fracture occurs. The stress peak is not very prominent and in fact

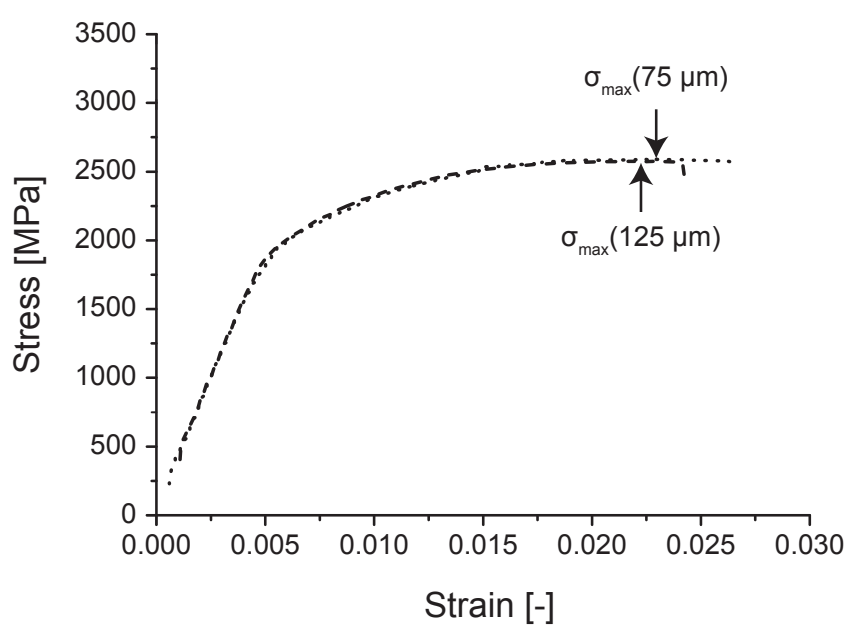

Figure 3: Stress-strain curves of two single fibre tension tests. The dotted curve correspond to a fibre with a diameter of $75 \mu \mathrm{m}$ the dashed curve to a fibre with a diameter of $125 \mu \mathrm{m}$. The curves are adapted in a way that the elastic line crosses the origin. The measuring length was approximately $15 \mathrm{~mm}$ in both cases. The maximum stress is indicated for both curves.

the deformation until fracture takes place at a more or less constant stress. The fracture stress is about $2500 \mathrm{MPa}$ with a failure strain of 0.024 and 0.027 respectively.

Figure 4 shows the force-displacement curve obtained from the tensile test on the single fibre composite. The response can be divided in three regimes. At first, the specimen deforms elastically following initial settlement of the system (region I). In the elastic regime, the applied load is mainly carried by the matrix, since the area ratio of matrix to fibre is 34 . At the maximum load of $252 \mathrm{~N}$ (corresponding to a maximum stress of $420 \mathrm{MPa}$ ), a crack extends in an uncontrolled manner over the whole cross section accompanied by a huge load drop (region II). The tomographic investigation, described in detail below, shows that in this region the fibre remains intact. After the matrix failure event, the fibre bears the full load. Under further displacement loading, the fibre is elongated plastically until ultimate fracture (region III).

Figure 5 shows cross-sections of tomograms at various displacement steps (numbers correspond to steps) after the matrix fracture (region III in Figure 4). In the upper row of the pictures the longitudinal cross-sections through the fibre centre position are shown. Picture no. 4 clearly reveals that the matrix is completely fractured. In addition, all cross-sections show plastic deformation and necking of the fibre. The matrix cracking is accompanied by interfacial debonding over a limited length. In the debonded domain, the applied load is concentrated in the fibre and thus the plastic deformation of the fibre is localized. Hence a large discrepancy between the nominal and true stresses applied to the fibre bridging a matrix crack is expected. With these cross-sections a quantitative determination of the extent of the necking (reduction of cross section area $A$ ) and the crack opening displacement $u$ is performed. The maximum crack opening $u_{\max }$ amounts to $90 \pm 2 \mu \mathrm{m}$, the crosssection area is reduced from $0.88 A_{0}$ at step 4 to $0.58 A_{0}$ at step 
4

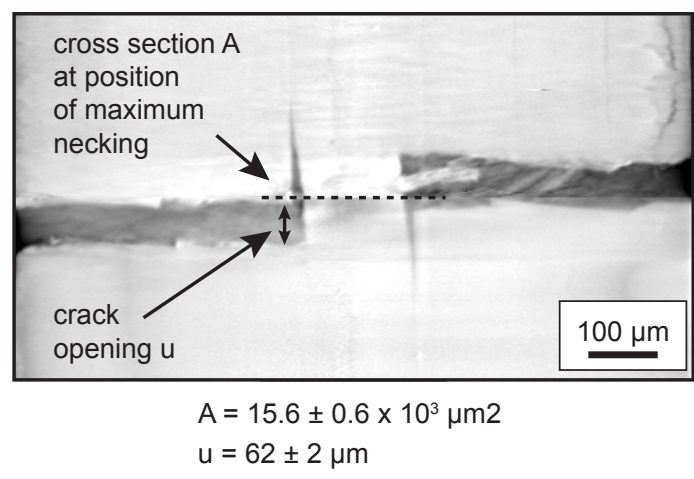

5

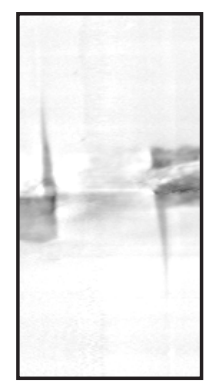

$15.0 \pm 0.6 \times 10^{3}$

$66 \pm 2$
6

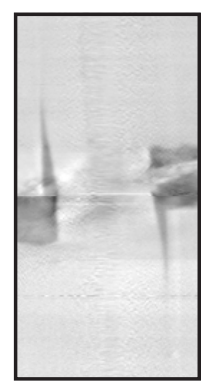

$14.3 \pm 0.6 \times 10^{3}$

$70 \pm 2$
7

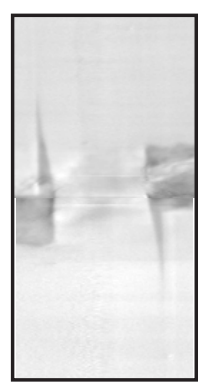

$13.3 \pm 0.6 \times 10^{3}$

$74 \pm 2$
8

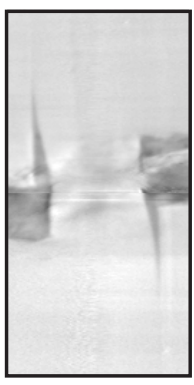

$12.7 \pm 0.6 \times 10^{3}$

$78 \pm 2$
9

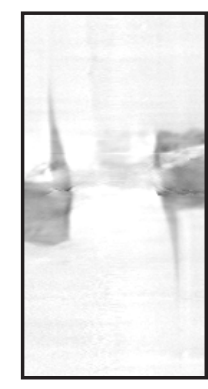

$11.1 \pm 0.5 \times 10^{3}$ $90 \pm 2$

Figure 5: Cross sections of tomograms showing the longitudinal views through the fibre centre at various displacement steps. Crack opening u and necking (reduction of cross section A) are determined from $2 \mathrm{D}$ cross sections. The displacement steps are indicated as numbers both in Figure 5 and 6 , where step 4 is immediately after the load drop event following the maximum force of $252 \mathrm{~N}$ (compare with Figure 4).

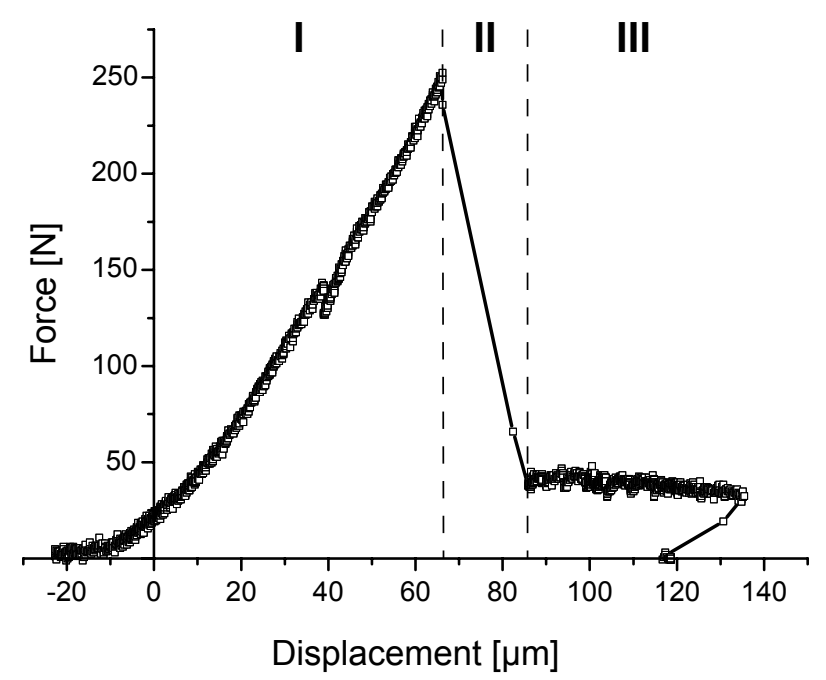

Figure 4: Force-displacement curve of a single fibre composite tension test. The curve is adapted in a way that the elastic line crosses the origin. Three regimes can be identified: elastic loading (region I), crack extension and load drop (region II) and uniform loading (region III). The load drop in region I was caused by relaxation as the displacment was stopped to record a tomogram.
9, where $A_{0}$ denotes the initial area. It has to be taken into account that the change in crack opening for adjacent displacement steps lies in the range of the spatial resolution however the overall change (no. 4 to no. 9) is obvious. The uncertainty in the lenght measurements shown in Figure 5 is taken equal to one voxel in the reconstructed image i.e. $2 \mu \mathrm{m} / p x$. This uncertainty has only minor influence on the later calculations and is therefore not taken into account for these.

The load and the nominal fibre stress are plotted in Figure 6 as open square symbols for the crack-bridging fibre after the matrix failure (region III in Figure 4). The nominal stress is based on the undeformed fibre cross section $A_{0}$. The line denotes a simple moving average with repeated boundaries (SMA) (500 points within 2000 data points). The vertical dashed lines indicate the displacement levels at which a tomographic measurement is performed. A slight stress relaxation occurs during the tomographic measurement. The true stress is plotted as circles based on the nominal (open circles) as well as on the true displacement (solid circles). The true stress and true displacement curve was calculated using the actual cross-section area and the crack opening displacement measured in the tomographic study. The true stress increases linearly to a value of $3200 \mathrm{MPa}$, which is higher than the nominal stress values by nearly $50 \%$ at the final loading stage.

The energy consumption by plastic deformation strongly depends on the development of a plastic zone and hence on the length of the debonded interface between fibre and matrix. The three-dimensional extent of interfacial debonding can be visualized by means of tomographic $3 \mathrm{~d}$ reconstructions.

Figure $7 \mathrm{a}$ shows the three-dimensional crack geometry reconstructed for three different viewing angles at displacement step 4. The two planes with rough surfaces indicate the flanks of the matrix crack being bridged by the fibre which is orientated perpendicular to the crack surfaces. The distance between the planes corresponds to the crack opening displacement $u$. The reconstruction image also shows the interfacial crack along the fibre segment which is debonded from the matrix. 
(a) volume reconstrution of crack in fibre area

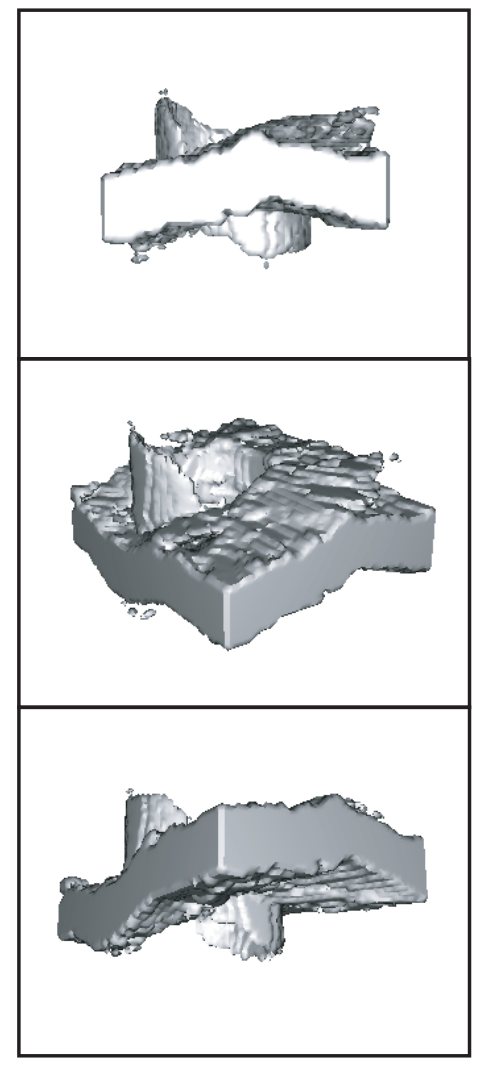

(b) interface debonding

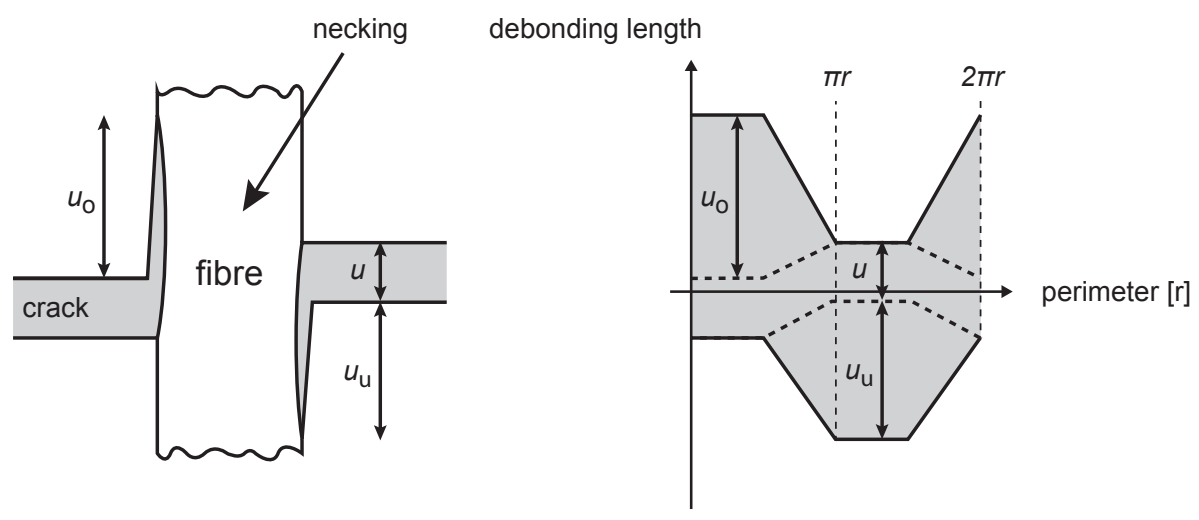

(c) debonding length
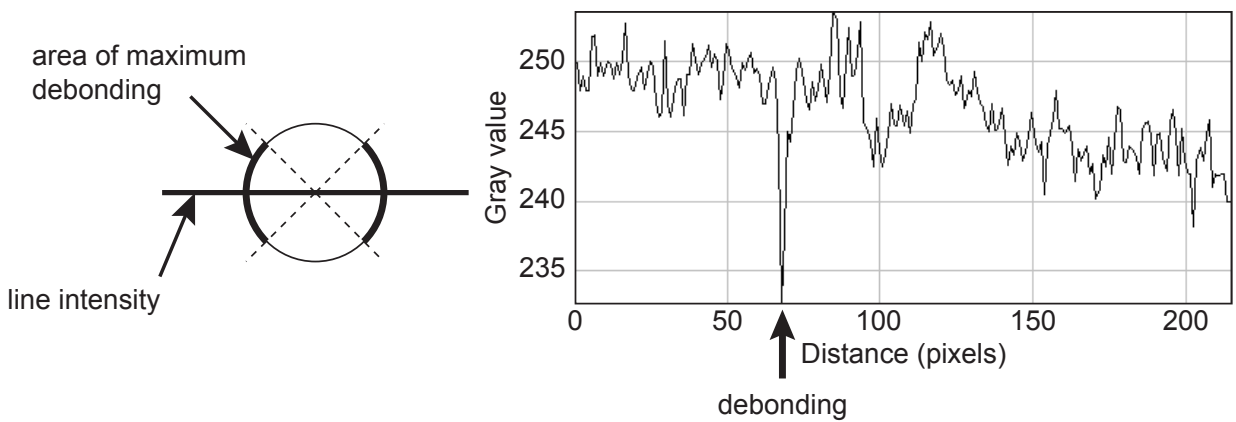

Figure 7: (a) Tomographic 3D reconstruction of the matrix crack bridged by an intact fiber. The 3D extend of debonding is clearly visible. (b) A projected schematic representation indicating the debonded area as a cross-section (left) and along the perimeter (right). (c): Grey level profile across the fibre expressed as gray scale values. The minimum in the intensity indicates debonding. 


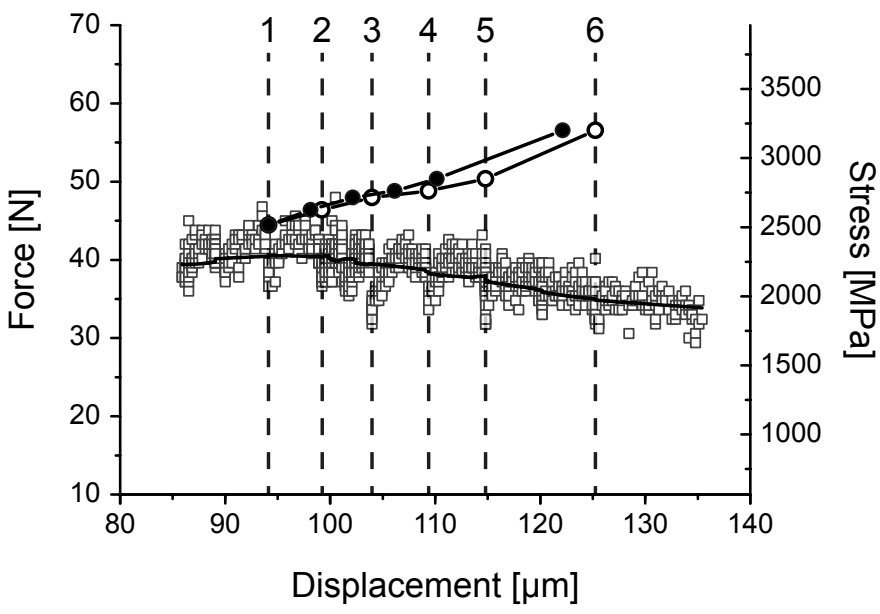

Figure 6: The load and the nominal fibre stress (based on the undeformed fibre cross section) are plotted as square symbols after matrix failure. The simple moving average of the fibre stresses is given as black line. The true stress is plotted as circle symbols over the nominal (open symbols) as well as the true displacement (solid symbols). The vertical dashed lines indicate the displacement levels at which a tomographic measurement is performed.

The geometry of Figure 7a is depicted schematically as a projected picture in Figure $7 \mathrm{~b}$. Clearly the debonding occurs on both sides of the crack, but incompletely along the half periphery. As some of signal information may possibly get lost by threshold setting for the grey scale filtering of tomographic data, the debonding length was determined using grey level profiles at different heights across the fibre. A typical line intensity plot is shown in Figure $7 \mathrm{c}$ where the minimum indicates debonding. Tomographic cross sections at different heights along the fibre are evaluated accordingly up to distances from the crack where no debonding is detected anymore. The debonding length $u_{\mathrm{db}}$ is determined from the crack opening distance.

The mean debonding length $u_{\mathrm{db}}$ (the average value of upper debonding $u_{\mathrm{db}, \mathrm{u}}$ and lower debonding $\left.u_{\mathrm{db}, 1}\right)$ is found equal to $54 \pm 2 \mu \mathrm{m}$ at step 4 and $66 \pm 2 \mu \mathrm{m}$ at step 9 , respectively. The area of debonded interface is $25.4 \pm 1.2 \times 10^{3} \mu \mathrm{m}^{2}$ and $31.1 \pm 1.2 \times 10^{3} \mu \mathrm{m}^{2}$, respectively. Assuming that the deformation of the fibre is concentrated in the debonded area, the fibre strain in that area can be expressed in terms of the crack opening $u$ as $\epsilon=u / u_{\mathrm{db}}$. The calculated strain is 1.15 in step 4 and 1.36 in step 9 . These values are very high compared to the conventional tensile test data of a single fiber where the fracture strain is normally about 0.02 .

After the in-situ tensile test at ESRF, the fractured surfaces are investigated by scanning electron microscopy (SEM). In Figure 8, the fracture surface of the ruptured specimen is shown in SEM images. The plastic elongation and necking deliver the clear evidence that the fibre experienced significant ductile deformation. The fracture surface of the fibre reveals a highly textured, fibrous microstructure, whereas the CVD matrix shows a mixture of cleavage and intergranular fracture. The cleavage and intergranular fracture observed in the matrix is character- istic for brittle tungsten [22], whereas the alveolar tearing is typical for drawn tungsten wire [23]. The fracture surface of the matrix crack is well aligned to the radial growth direction of the matrix.

As the detached fibre continues to plastically deform leading to necking, the bundle of long grains in the fibre with low grain boundary strength [24] begins to experience a tri-axial stress state and undergoes intergranular debonding. The process takes place over a small region while the bonding in grain areas located beyond this region remains still intact. The individual grains being detached from each other act now as if they were stand-alone monocrystalline fibres having high ductility. During continued tensile loading, they neck down to a single point.

\section{Quantitative assesment of toughening effect}

The plastic behaviour of an embedded fibre such as flow stress and failure strain depends strongly on the local mechanical constraints (interface strength, friction, residual stress etc.) [11]. The measured strain in the debonded region is more than $100 \%$ which seems extraordinarily large compared to the case of the conventional tensile test on the free fibres where the ultimate strain ranges between 2-3\% (compare Figure 3). On the other hand, the measured true stress is of the same order and comparable to the typical tensile strength given in literature (2$4 \mathrm{GPa}$ [24]). The total applied work $W_{\mathrm{f}}$ consumed by elastic and plastic deformation of the fibre can be approximated as a product of mean force $\bar{F}$ (obtained from the curve in Figure 6) and the total displacement which is equal to crack opening $u$, that is,

$$
W_{\mathrm{f}}=\bar{F} \cdot u=38 \mathrm{~N} \cdot 90 \mu \mathrm{m}=3.42 \mathrm{~J}
$$

Using the elastic modulus of tungsten $E=400 \mathrm{GPa}$ the fraction of elastic strain energy is calculated to be smaller than $1 \%$ and hence most of the work $W_{\mathrm{f}}$ is predominately used for plastic deformation. The specific plastic deformation energy $w_{\mathrm{f}, \mathrm{pl}}^{*}$ per unit area of the fibre is calculated by considering the actual dimension of the fibre.

$$
w_{\mathrm{f}, \mathrm{pl}}^{*}=\frac{W_{\mathrm{f}}}{r_{\mathrm{f}}^{2} \pi}=0.19 \mathrm{~J} \mathrm{~mm}^{-2}
$$

The energy density $\tilde{w}_{\mathrm{f}}$ per unit volume of the fibre (based on a length of $1 \mathrm{~mm}$ ) is then expressed as follows:

$$
\tilde{w}_{\mathrm{f}, \mathrm{pl}}=0.19 \mathrm{~J} \mathrm{~mm}^{-3}
$$

Using the empirical equations suggested Ashby et al. 2 and 3 for calculating $w_{\mathrm{f}, \mathrm{pl}}^{*}$ yields:

$$
\chi_{\mathrm{w}}=\frac{w_{\mathrm{f}, \mathrm{pl}}^{*}}{\sigma_{\mathrm{e}} r_{\mathrm{f}}}=1.5
$$

and therefore equation 3 adopts to

$$
\chi_{\mathrm{w}}=\frac{w_{\mathrm{f}, \mathrm{pl}}^{*}}{\sigma_{\mathrm{e}} r_{\mathrm{f}}}=1.25 \cdot \frac{u_{\mathrm{max}}}{r_{\mathrm{f}}}
$$




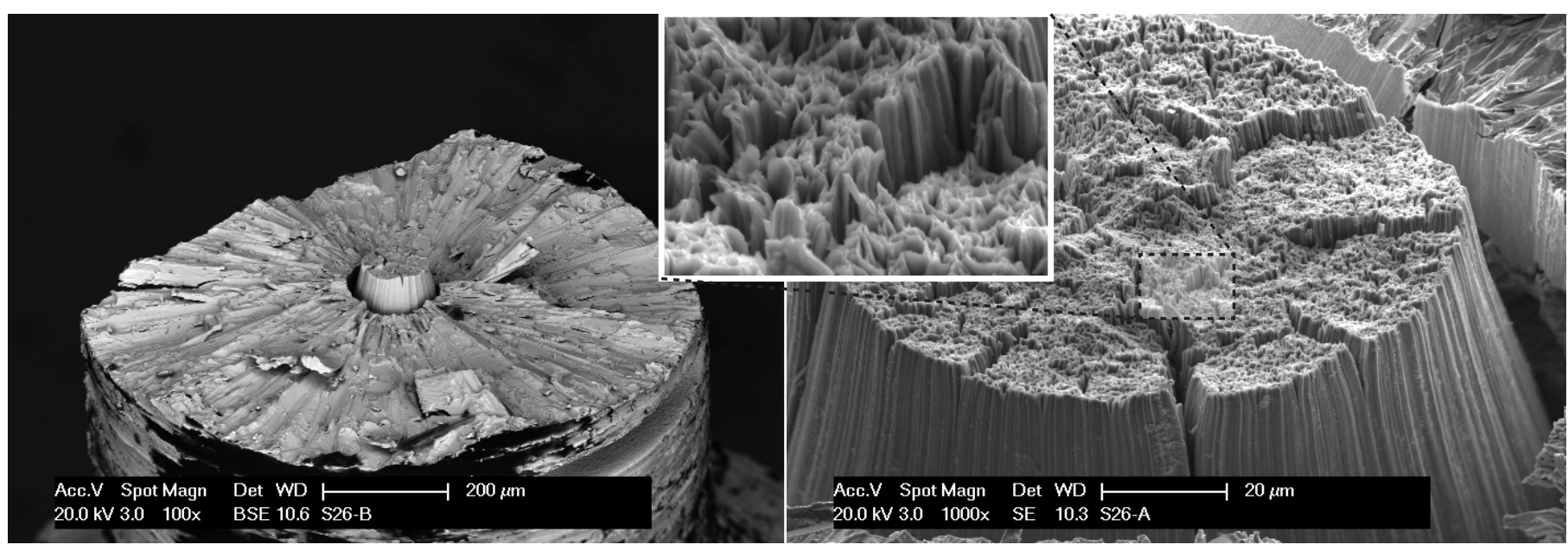

Figure 8: Fractography images of a broken single-fibre composite after tensile rupture (scanning electron microscopy).

in which $\chi_{\mathrm{w}}$ is the toughening parameter for the tungsten fibre, $u_{\max }=90 \mu \mathrm{m}$ is the maximum crack opening (displacement step 9) and $\sigma_{\mathrm{e}}=1700 \mathrm{MPa}$ is the yield strength of the tungsten fibre (see Figure 3). Except the slightly smaller gradient in the linear relation the tungsten composite seems to follow the same rules as suggested by Ashby et al. The difference might be attributed to a less prominent strain hardening effect in the tungsten fibre compared to the lead fibres used by them. The ratio of $\sigma_{\max }$ to $\sigma_{\mathrm{e}}$, which can be seen as a measure of strain hardening, is 1.2 for the tungsten fibre while it amounts to 3-6 for the lead fibres. The small work hardening rate of the tungsten fibre is due to its already strain hardened state which is a consequence of the drawing process during production. In contrast, the lead fibres were produced by melt infiltration and thus have initially a reduced dislocation density, which is a prerequisite for strong work hardening. The adopted Ashby-equations can be used as a first approximation of the plastic deformation energy in $W_{\mathrm{f}} / W$-composites.

The influence of the constraint on the the plastic deformation energy and thus on the overall toughening potential can be evaluated by looking on the results of the single-fibre tension tests on the free fibres. The specific deformation energy is often calculated using the stress-strain curve measured in such single-fibre tension tests (sft), e.g. Pemberton et al. [25]. In the case of the tungsten wire, the plastic deformation energy $w_{\mathrm{f}, \mathrm{pl} \text {,sft }}$ by unit volume is calculated to be $0.054 \mathrm{~J} \mathrm{~mm}^{-3}$. This value is obtained by integrating the stress-strain curve (mean value of curves given in Figure 3) and can be compared with the values of the constraint case by scaling with the maximum measured debonding length $u_{\mathrm{db}, \max }=66 \pm 2 \mu \mathrm{m}$.

$$
\begin{aligned}
& w_{\mathrm{f}, \mathrm{pl}, \mathrm{sft}}^{*}=w_{\mathrm{f}, \mathrm{pl}, \mathrm{stt}} \cdot u_{\mathrm{db}, \max }=0.0036 \mathrm{~J} \mathrm{~mm}^{-2} \\
& \tilde{w}_{\mathrm{f}, \mathrm{pl}, \mathrm{st}}=0.0036 \mathrm{~J} \mathrm{~mm}^{-3}
\end{aligned}
$$

The resulting value is much smaller than the value determined in the constraint case. The reason for that is that in integrating the stress-strain curve a homogenous behaviour over the whole fibre length is assumed. In reality this is of course not true, as a huge part of the plastic deformation is locally concentrated in a small area because of necking. This approach, which assumes uniform straining of the fibre, is therefore not adequate for determining the plastic deformation energy in a composite as already pointed out by Ashby et al. [11].

Nevertheless a comparison of the deformation energy consumed by necking in a single-fibre test and in a constraint case can give an indication about the "quality" of the composite. The plastic deformation energy per unit length is much larger in the necking zone than in the rest of the fibre. Debonding of a length similar the size of the necking zone allows therefore a most effective energy dissipation by ductile fibre deformation. For the tungsten fibre, an approximate value of this energy can be derived from the curve shown in Figure 3. Assuming that the maximum stress is the starting point of necking the mean plastic deformation energy in the necking zone can be approximately determined by integrating the stress-displacement curve. In the case of Figure $3 w_{\mathrm{f}, \mathrm{pl} \text {, stt,necking }}=0.093 \mathrm{~J} \mathrm{~m}^{-2}$ for a displacement in the necking zone of $40 \mu \mathrm{m}$. This value is of course much higher than the one obtained by assuming a uniform elongation of the fibre, but also, more importantly, it is of the same order of magnitude as the value determined by the tomography experiment (constraint case). In practice it means that the debonding length obtained in the system considered here is almost sufficient to dissipate the plastic deformation energy available in the fibre most effective. For a more detailed comparison a sophisticated understanding of the formation, growing and extend of the necking zone is important and therefore further investigations are needed to get an deeper understanding of the effective mechanism.

Table 1 summarizes the results for the plastic deformation energy obtained for the constraint and non-constraint case. Our investigation manifests the importance of the constraint with 
Table 1: Summary of different approaches for determining the plastic deformation energy

\begin{tabular}{|c|c|c|c|}
\hline & $\begin{array}{c}\text { win } \\
{\left[\mathrm{J} \mathrm{mm}^{-3}\right]}\end{array}$ & \multicolumn{2}{|c|}{ constraint } \\
\hline $\begin{array}{l}\mathrm{W}_{\mathrm{f}, \mathrm{pl}} \\
\text { force-displ. }\end{array}$ & 0.19 & $66 \mu \mathrm{m}$ & constraint \\
\hline $\begin{array}{l}\mathrm{W}_{\mathrm{f}, \mathrm{pl}, \mathrm{sft}} \\
\text { stress-strain }\end{array}$ & 0.0036 & & $\begin{array}{l}\text { no constraint } \\
\text { uniform } \\
\text { deformation }\end{array}$ \\
\hline $\begin{array}{l}\mathrm{W}_{\mathrm{f}, \mathrm{pl} \text {,sft,necking }} \\
\text { stress-displ. } \\
\text { necking zone }\end{array}$ & 0.093 & & $\begin{array}{l}\text { no constraint } \\
\text { necking }\end{array}$ \\
\hline
\end{tabular}

Table 2: Extrinsic toughening in ceramics after [14]

\begin{tabular}{cc}
\hline Mechanism & Highest toughness in $\left[\mathrm{MPa} \mathrm{m}^{0.5}\right]$ \\
\hline Transformation & $\sim 20$ \\
Microcracking & $\sim 10$ \\
Metal dispersion & $\sim 25$ \\
Whiskers/platelets & $\sim 15$ \\
Fibres & $\leq 30$ \\
\hline
\end{tabular}

regard to the toughening by plastic deformation. Information on the exact extent of the debonding and thus the zone of plastic deformation is very important to be able to approximate the amount of plastic deformation energy. Single-fibre tension tests (no constraint) with a gauge length comparable to the actual debonding length can provide the proper values for estimating the contribution to the toughening. On the other hand, the extent of the plastic zone determined in a single-fibre test gives an idea for an optimal debonding length.

Assuming a fibre-volume fraction of $30 \%$ and a full contribution of each fibre, the contribution of plastic deformation to the extrinsic toughening can be estimated using equation 1 as

$$
\begin{aligned}
& \Delta G_{\mathrm{pl}}=5.7 \pm 0.1 \times 10^{4} \mathrm{~J} \mathrm{~m}^{-2} \\
& \Delta K_{\mathrm{pl}}=\sqrt{\Delta G_{\mathrm{pl}} E}=151 \pm 2 \mathrm{MPa} \mathrm{m}^{0.5}
\end{aligned}
$$

\section{with $\quad K:$ Stress intensity factor $\left[\mathrm{MPa} \mathrm{m}^{0.5}\right]$

$$
E \text { : Young's Modulus [400 GPa] }
$$

For comparison, the toughening effect originating from several different mechanisms given by Evans et al. are listed in Table 2 [14]. In comparison to these values the toughness enhancement based on the plastic deformation of the tungsten wire is relatively high. Although assuming a full contribution by each fibre represents an ideal case it reveals the high potential of the mechanism.

\section{Conclusion and outlook}

To the best of the authors knowledge this is the first time that the toughening by plastic fibre deformation is determined directly on a real composite system rather than a model system. The contribution to the toughness of tungsten fibre reinforced tungsten composites has been investigated by means of mechanical tests in combination with in-situ high energy synchrotron tomography. Tension tests on a single fibre composite system allows the quantitative estimation of the plastic deformation contribution to the extrinsic toughening. The main conclusion is, provided that there is adequate interface debonding (range: $50-100 \mu \mathrm{m}$ ), the plastic deformation gives significant contribution to the toughening in $W_{\mathrm{f}} / W$-composites. Only the in-situ tomographic observation allows to get this information as it gives full insight into all relevant contributions as e.g. the constraint (debonding) or the crack opening. The formation and behaviour of the necking zone has a strong influence on the plastic deformation energy.

In the future the role of the necking in the plastic deformation of the tungsten fibre will be determined by single fibre tension tests. Therefore tests with different measuring lenghts and strain rates are going to be performed on fibres with different diameters. In microscopic investigatons the size of the necking zone will be evaluated. This will allow the optimization of composite itself. Tension tests on samples with a fibre volume fraction of $30 \%$ will allow a verification of the estimated toughening contribution by the plastic fibre deformation.

\section{Acknowledgements}

The authors want to thank J. Du, M. Köppen and A. Zivelonghi who contributed substantially by their experimental assistance to the success of this investigation. We also acknowledge support by Osram GmbH, Schwabmünchen, Germany for providing the tungsen wire and Archer Technicoat Ltd, High Wycombe, UK for the CVD production. For their assistance in applying and setting up a tomography experimental campaign we want to thank the Riso National Lab and representative Soeren Faester Nielen and Henning Fries Poulsen. This work was supported by EURATOM and was partly carried out within the Seventh Framework Programme (Grant Agreement 224752). The views and opinions expressed herein do not necessarily reflect those of the European Commission.

\section{References}

[1] Baluc N. Final report on the EFDA task TW1-TTMA-002 deliverable 5. Tech. Rep.; Centre de Recherches en Physique de Plasmas; 2002.

[2] You JH, Komarova I. Probabilistic failure analysis of a water-cooled tungsten divertor: Impact of embrittlement. Journal of Nuclear Materials 2008;375:283-9.

[3] Rieth M, Dudarev S, Gonzalez de Vicente S, Aktaa J, Ahlgren T, Antusch $\mathrm{S}$, et al. Recent progress in research on tungsten materials for nuclear fusion applications in europe. Journal of Nuclear Materials 2013;432:482500 .

[4] Wurster S, Baluc N, Battabyal M, Crosby T, Du J, Garca Rosales $\mathrm{C}$, et al. Recent progress in $\mathrm{R} \& \mathrm{D}$ on tungsten alloys for divertor structural and plasma facing materials. Jounal of Nuclear Materials 2013; doi:10.1016/j.jnucmat.2012.02.074; in press. 
[5] Du J, Höschen T, Rasinski M, Wurster S, Grosinger W, You JH. Feasibility study of a tungsten wire reinforced tungsten matrix composite with $\mathrm{ZrO}_{\mathrm{x}}$ interfacial coatings. Composites Science and Technology 2010;70:1482-9.

[6] Du J, Höschen T, Rasinski M, You JH. Interfacial fracture behavior of tungsten wire/tungsten matrix composites with copper-coated interfaces. Materials Science and Engineering: A 2010;527:1623-9.

[7] Riesch J, Höschen T, Galatanu A, You JH. Tungsten-fibre reinforced tungsten composites: A novel concept for improving the toughness of tungsten. In: Proceedings of the 18th International Conference on Composite Materials. Jeju, South Korea; 2011,.

[8] Riesch J. Entwicklung und Charakterisierung eines wolframfaserverstärkten Wolfram-Verbundwerkstoffs. Ph.D. thesis; Technische Universität München; 2012.

[9] Chawla K. Ceramic matrix composites. Chapman \& Hall; 1993.

[10] Jones R, Henager Jr. C. Subcritical crack growth processes in SiC/SiC ceramic matrix composites. Journal of the European Ceramic Society 2005;25:1717-22.

[11] Ashby M, Blunt F, Bannister M. Flow characteristics of highly constrained metal wires. Acta Metallurgica 1989;37:1847-57.

[12] Murphy J, Giannattasio A, Yao Z, Hetherington C, Nellist P, Roberts S. The mechanical properties of tungsten grown by chemical vapour deposition. Journal of Nuclear Materials 2009;386-388:583-6.

[13] Anderson T. Fracture Mechanics: Fundamentals and Applications. Taylor \& Francis Group LLC; 2005.

[14] Evans A. Perspective on the development of high-toughness ceramics. Journal of the American Ceramic Society 1990;73:187-206.

[15] Du J. A feasibility study of tungsten-fiber-reinforced tungsten composites with engineered interfaces. Ph.D. thesis; Technische Universität München; 2011.

[16] Buffière JY, Cloetens P, Ludwig W, Maire E, Salvo L. In situ X-ray tomography studies of microstructural evolution combined with $3 \mathrm{~d}$ modelling. MRS Bulletin 2008;33:611-9.

[17] Riesch J, Linsmeier C, Nielsen SF. In-situ tomographic observation of crack formation and propagation in tungsten materials in the framework of FEMaS-CA. In: Challenges in materials science and possibilities in 3D and 4D characterization techniques - Proceedings of the 31st Ris International Symposium on Materials Science. Roskilde, Denmark; 2010, p. 405-12.

[18] Zivelonghi A. Thermomechanical behaviour of two heterogeneous tungsten materials via $2 \mathrm{~d}$ and $3 \mathrm{~d}$ image-based FEM. Ph.D. thesis; Technische Universität München; 2011.

[19] Martin T, Koch A. Recent developments in X-ray imaging with micrometer spatial resolution. Journal of Synchrotron Radiation 2006;13(Part 2):180-94.

[20] ImageJ . rsbwebnihgov/ij/ 2011;.

[21] Buffière JY, Maire E, Cloetens P, Lormand G, Fougres R. Characterization of internal damage in a MMCp using X-ray synchrotron phase contrast microtomography. Acta Materialia 1999;47:1613-25.

[22] Pippan R, Wurster S, Baluc N, Battabyal M, Crosby T, Du J, et al. Recent progress in $\mathrm{R} \& \mathrm{D}$ on tungsten alloys for divertor structural and plasma facing components. Charlston, USA: 15th International Conference of Fusion Reactor Materials (ICFRM); 2011,

[23] Leber J, Tavernelli J, White D, Hehemann R. Fracture modes in tungsten wire. Journal of Less-Common Metals 1976;48:119-33.

[24] Lassner E, Schubert WD. Tungsten - Properties, Chemistry, Technology of the Element, Alloys, and Chemical Compounds. Kluwer Academic / Plenum Publishers; 1999.

[25] Pemberton S, Oberg E, Dean J, Tsarouchas D, Markaki A, Marston L, et al. The fracture energy of metal fibre reinforced ceramic composites (MFCs). Composites Science and Technology 2011;71:266-75. 